\title{
Optimization of Parametric Comb Generation Using Interferometric Wavelength Selective Switch
}

\author{
Mohammad A. Z. Al-Khateeb ${ }^{(1)}$, Mary E. McCarthy ${ }^{(1)}$, M. Deseada Gutierrez Pascual ${ }^{(2)}$, Frank Smyth ${ }^{(2)}$, \\ Andrew D. Ellis ${ }^{(1)}$ \\ ${ }^{(1)}$ Aston Institute of Photonic Technology, Aston University, Aston Triangle, Birmingham, UK \\ ${ }^{(2)}$ Pilot Photonics, Invent Centre, Dublin City University, Glasnevin, Dublin 9, Ireland \\ Authore-mail address: alkhamaz@aston.ac.uk
}

\begin{abstract}
We propose and demonstrate frequency comb regeneration using parametric mixer dispersion managed by interferometric wavelength selective switch. The results show a good control over the bandwidth/flatness of the comb generated by the parametric process.

OCIS codes: (190.4380) Nonlinear optics, four-wave mixing; (190.4410) Nonlinear optics, parametric processes.
\end{abstract}

\section{Introduction}

Coherent optical transmission and wavelength division multiplexing (WDM) are accepted to be significant technologies to enable capacity expansion of optical communication systems by enabling higher order modulation formats, superchannels, and flex-grid WDM. To achieve performance stability of coherent higher order modulation formats, the operator should deploy local oscillator (LO) lasers that have low linewidth, low relative intensity noise (RIN), low spectral and phase instability in WDM grid lines. Optical frequency combs have been proposed as LO source in optical transmission systems [1] to achieve system-wide coherency (that can allow full-field digital backpropagation [2]) and reduce deployed recourses in the systems (i.e. using single comb source instead of dedicated laser for each WDM channel). An optical frequency comb should originate from a single, low linewidth, source seed to guarantee phase locking of the generated comb lines. Also, it should realize stable (and preferably flexible) frequency spacing between the generated lines. One way of comb generation is using parametric nonlinear process in optical fiber (namely four wave mixing FWM) [3] seeded by phase locked laser lines, this methodology needs a very careful dispersion engineering through the different stages of mixers to generate flat comb lines (theoretically described in [4]). A pre-engineered dispersion management in multistage parametric comb generator will limit the flexibility of the comb to change the frequency spacing between its lines.

In this paper, we propose and demonstrate the use of interferometric wavelength selective switch (WSS) and a parametric mixer for achieving comb regeneration, showing that the WSS can provide flexible pre-dispersion management to control the outcome of the parametric process to generate comb. By optimizing the phases of comb source lines (flat 13 lines with $10 \mathrm{GHz}$ frequency separation), we have been able to almost triple the number of comb lines (keeping $1 \mathrm{~dB}$ flatness) generated by a single stage parametric nonlinear mixer.

\section{Experimental setup and results}

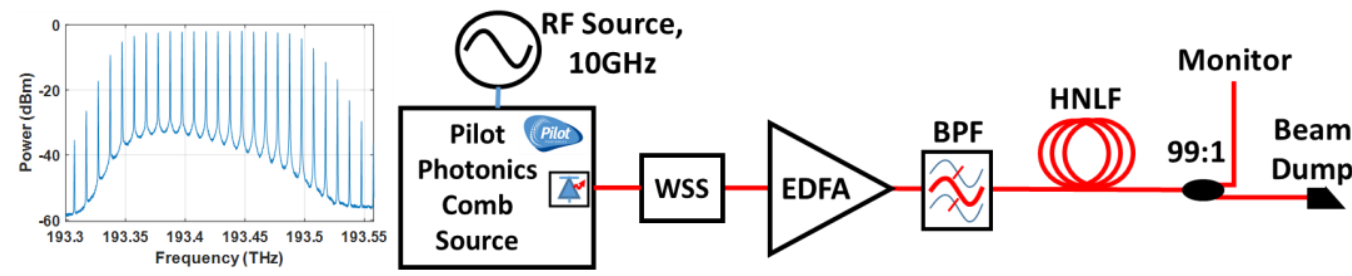

Fig. 1 Parametric comb regeneration: (left) optical spectrum generated by pilot photonics comb source, (right) experimental setup.

In order to evaluate the capabilities of comb regeneration (pre-dispersed by interferometric WSS), we implemented the experimental setup shown in Fig. 1 (right). The seed of the parametric comb regenerator is generated using a Pilot photonics comb source [5] centered at $1550 \mathrm{~nm}$. The seed consists of 13 lines (separated by $10 \mathrm{GHz}$ ) with $1 \mathrm{~dB}$ of power variation (Fig. 1 (left)). Next a programmable/interferometric WSS with a resolution of $1 \mathrm{GHz}$ is used to control the phases of output comb lines to emulate dispersion. The output of WSS is amplified by high power EDFA to $33 \mathrm{dBm}$ and filtered by a band pass filter (BPF) to eliminate the out-of-band amplified spontaneous emission (ASE) noise generated by the EDFA. The filtered $13 \mathrm{comb}$ lines (with a total power of $31.5 \mathrm{dBm}$ ) are propagated through a $70 \mathrm{~m}$ highly nonlinear fiber (HNLF) that has a zero dispersion wavelength (ZDF) at $1551 \mathrm{~nm}$, nonlinear factor of 21.4(W.km) ${ }^{-1}$, dispersion slope of $0.043 \mathrm{ps} / \mathrm{nm}^{2} / \mathrm{km}$, stimulated Brillouin scattering (SBS) threshold of $22 \mathrm{dBm}$. The regenerated comb (at the output of HNLF) was monitored, at $1 \%$ output, by $150 \mathrm{MHz}$ resolution optical spectrum analyzer (OSA). 


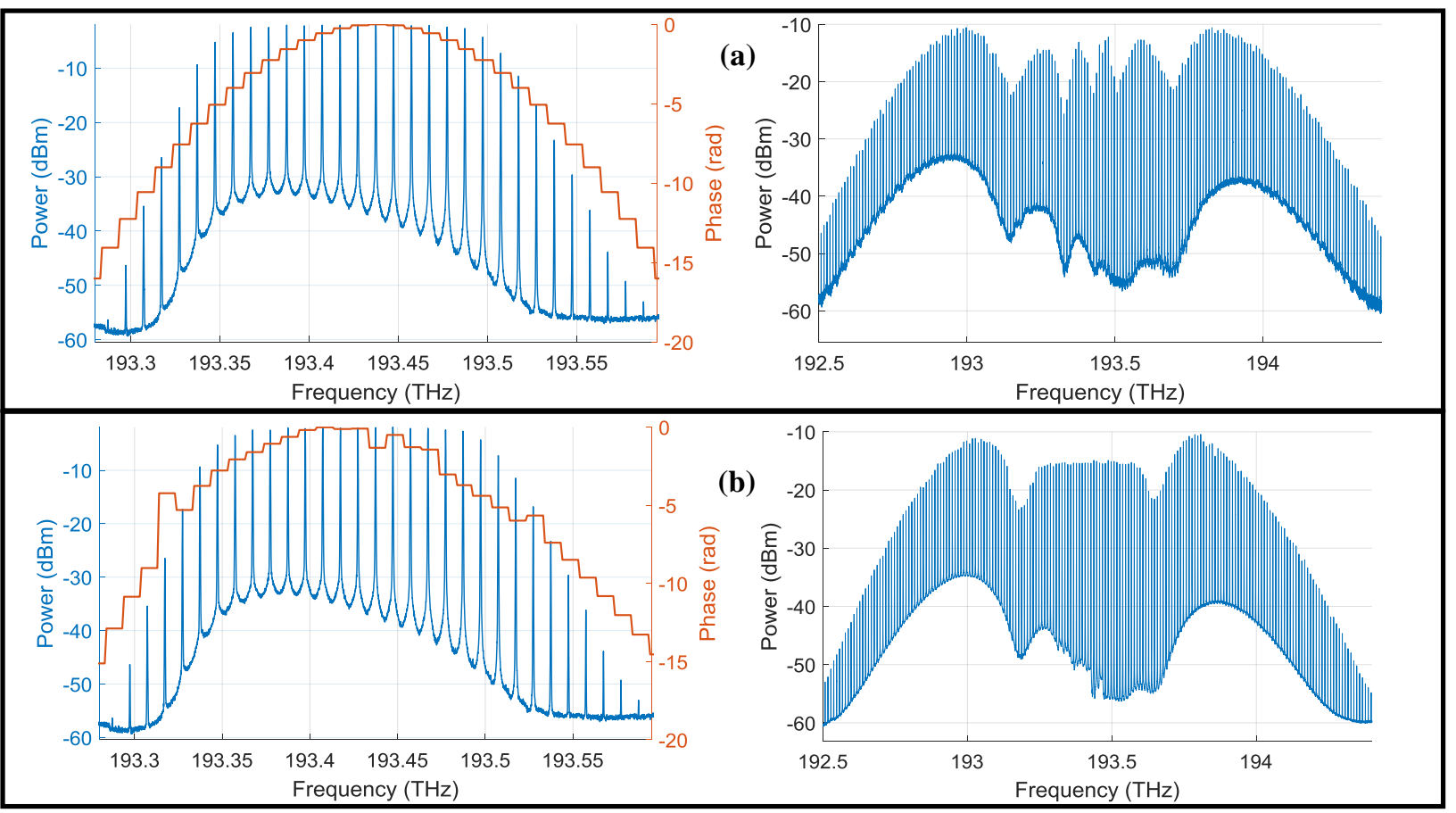

Fig. 2 (a) left plot shows that comb source output and the phase profile applied to its lines by WSS, the right plot shows the regenerated comb at the output of HNLF. (b) left plot shows that comb source output and the phase profile applied to its lines by WSS, the right plot shows the regenerated comb at the output of HNLF.

As we have applied an equivalent of $-25 \mathrm{ps} / \mathrm{nm}$ dispersion to the source comb lines (see fig. 2(a)), we have seen that the comb lines will be regenerated to spread over $1.4 \mathrm{THz}$ with power variation of $14 \mathrm{~dB}$ and a line extinction ratio ranging from $25 \mathrm{~dB}$ to $38 \mathrm{~dB}$ (source extinction ratio ranges from $28 \mathrm{~dB}$ to $44 \mathrm{~dB}$ for comparison). By optimizing the individual phases of comb source lines to achieve the $1 \mathrm{~dB}$ flatness in regenerated comb (see fig. 2(b)), we were able to achieve 38 lines ( $380 \mathrm{GHz}$ bandwidth $/ 10 \mathrm{GHz}$ spacing) and line extinction ratio ranging from $26 \mathrm{~dB}$ to $40 \mathrm{~dB}$. From fig. $2(\mathrm{a} \& \mathrm{~b})$, it can be seen that the side lopes of the regenerated comb cannot be eliminated since they are resulted from higher order FWM resulted at a phase mismatching state according to the local dispersion of the HNLF (due to the high dispersion slope of our fiber). The parametric ASE noise (modulation instability) floor variation between the side lopes can be explained by the fact that lower frequency side lope is resulted from the FWM at normal HNLF local dispersion (D>0), while the higher frequency side lope is resulted from the FWM at negative HNLF local dispersion $(\mathrm{D}<0)[4]$.

\section{Conclusion}

We experimentally demonstrated that introducing an interferometric WSS pre-dispersing comb seed lines can flexibly change parametric nonlinear interaction in an optical mixer medium. The experiment conducted in this work, shows that the number of regenerated comb lines can be, at least, triple the bandwidth of a seed comb source with a restricted requirement of power flatness.

Acknowledgment: this work was partially supported by the EPSRC via projects EP/J017582/1 and EP/L000091/1 (UNLOC and PEACE), Original data for this work is available through Aston Research Explorer (http://doi.org/10.17036/researchdata.aston.ac.uk.00000174).

\section{References}

[1] D. Hillerkuss, et al., "26 Tbit s-1 line-rate super-channel transmission utilizing all-optical fast Fourier transform processing," Nat. Photonics 5, 364-371 (2011)

[2] S. Randel, et al., "Fiber Nonlinearity Compensation by Digital Backpropagation of an Entire 1.2-Tb/s Superchannel Using a Full-Field Spectrally-Sliced Receiver," in 39th European Conference and Exhibition on Optical Communication (ECOC 2013) (Institution of Engineering and Technology, 2013), pp. 48-50.

[3] G. A. Sefler and K.-I. Kitayama, "Frequency comb generation by four-wave mixing and the role of fiber dispersion," J. Light. Technol. 16, 1596-1605 (1998).

[4] Evgeny Myslivets, et al., "Generation of wideband frequency combs by continuous-wave seeding of multistage mixers with synthesized dispersion," Opt. Express 20, 3331-3344 (2012).

[5] http://www.pilotphotonics.com/PP-OWCS.pdf . 